\title{
Topical issue on Multi-scale phenomena in complex flows and flowing matter
}

\author{
Published online: 27 May 2016 - (C) EDP Sciences / Società Italiana di Fisica / Springer-Verlag 2016
}

\begin{abstract}
New research topics related to the statistical and dynamical properties of matter flowing in simple and complex flows have emerged in the recent years at the crossroads among industrial processes, biotech applications, fundamental physics, engineering and Earth sciences. Depending on the microscopic interactions, a sample of molecules or mesoscopic particles can flow like a simple Newtonian fluid, deform elastically like a solid, or behave with a complex rheology and strong non-equilibrium properties. When the internal constituents are active, as for biological entities, complicate collective motions can be observed. Sometimes these particles undergo breakup or coalescence processes, whose occurrence strongly depends on the flow statistics.

In many realistic applications, the large scale advecting flow has chaotic spatial and temporal fluctuations, sometimes over a wide range of scales/frequencies as in the case of fully developed turbulent flows. As a result, flowing matter often exhibits a tight coupling between small-scale fluctuations and large-scale flow structures, urging for a unifying view at both Lagrangian and Eulerian levels.

Flows of interest can be at the nano-, micro- or macro-scales, and need different theoretical, numerical and experimental approaches. The interplay of the dynamical and other related processes taking place at the different scales remains, for many problems, elusive and challenging.

This topical issue of the European Physical Journal E brings together a cross-section of research advances and activities, highlighting the diversity of basic and applied interests in flowing matter across the scales. It is the result of a widespread activity in Europe and beyond, involving communities from physics, bio-physics, mechanical engineering and applied mathematics.

Several papers discuss peculiar aspects of complex flowing behaviour at changing the characteristic lengthscales of the system, in particular focusing on thermal fluctuations, nanoscale heterogeneities and structural transition [1-5]. The discrete nature of the dispersed phase is investigated in terms of Lagrangian structures at micro/meso-scale (e.g. colliding particles, polymers, active matter, micro-swimmers) by several authors [6-11]. Few contributions focus on classical problems related to non-linear systems or the turbulent behaviour of Newtonian fluids, but focusing on new observables or novel approaches to anomalous scaling: these include the discussion of dissipated energy in diffusive systems [12], turbulence under rotation or with strong helical properties [13-16], with waves [17], or with modified non-linear interactions $[18,19]$.

The papers in this issue showcase examples of active mutual scientific fertilization across different disciplines and approaches. Among these, the application of techniques inherited from statistical mechanics, both in analyzing largescale dynamics of chaotic systems, as well as mesoscale statistics of glass-forming liquids, but also energy-landscape analysis of mechanical deformation and the study of flowing properties in jammed systems, just to name a few. The ultimate goal of this compilation is to further stimulate the interaction across the boundaries between fundamental and applied research, and among scientists of different areas, leading to the emergence of new challenging directions, bold concepts, and exciting developments.
\end{abstract}

We acknowledge support from COST Action MP1305, the European Research Council under the EU Seventh Framework Programme (FP7/2007-2013) / ERC Grant Agreements no 279004 and no. 339032. Some of the contributing authors took part in the Conference "Flowing Matter Across the Scales" held in Rome (Italy), from March 24 to March 27, 2015, which inspired this Special Issue. 


\section{References}

1. Anupam Gupta, Mauro Sbragaglia, Eur. Phys. J. E 39, 6 (2016) "Effects of viscoelasticity on droplet dynamics and break-up in microfluidic T-Junctions: a lattice Boltzmann study", DOI: 10.1140/epje/i2016-16006-9.

2. Anupam Gupta, Mauro Sbragaglia, Eur. Phys. J. E 39, 2 (2016) "A lattice Boltzmann study of the effects of viscoelasticity on droplet formation in microfluidic cross-junctions", DOI: 10.1140/epje/i2016-16002-1.

3. Marcello Sega, Mauro Sbragaglia, Luca Biferale, Sauro Succi, Eur. Phys. J. E 38, 127 (2015) "The importance of chemical potential in the determination of water slip in nanochannels", DOI: 10.1140/epje/i2015-15127-y.

4. S. Kumar Ranjith, Eur. Phys. J. E 38, 89 (2015) "Mesoscopic simulation of single DNA dynamics in rotational flows", DOI: 10.1140/epje/i2015-15089-0.

5. Minh Triet Dang, Dmitry Denisov, Bernd Struth, Alessio Zaccone, Peter Schall, Eur. Phys. J. E 39, 44 (2016) "Reversibility and hysteresis of the sharp yielding transition of a colloidal glass under oscillatory shear", DOI: 10.1140/epje/i2016-16044-3.

6. Francesco Bonelli, Giuseppe Gonnella, Adriano Tiribocchi, Davide Marenduzzo, Eur. Phys. J. E 39, 1 (2016) "Spontaneous flow in polar active fluids: the effect of a phenomenological self propulsion-like term", DOI: 10.1140/epje/i2016-16001-2.

7. Kristian Gustavsson, Bernhard Mehlig, Eur. Phys. J. E 39, 55 (2016) "Statistical model for collisions and recollisions of inertial particles in mixing flows", DOI: 10.1140/epje/i2016-16055-0.

8. Mehdi Bouzid, Adrien Izzet, Martin Trulsson, Eric Clément, Philippe Claudin, Bruno Andreotti, Eur. Phys. J. E 38, 125 (2015) "Non-local rheology in dense granular flows - Revisiting the concept of fluidity", DOI: 10.1140/epje/i2015-15125-1.

9. Benjamin Dollet, Claire Bocher, Eur. Phys. J. E 38, 123 (2015) "Flow of foam through a convergent channel", DOI: 10.1140/epje/i2015-15123-3.

10. Silvia Varagnolo, Giampaolo Mistura, Matteo Pierno, Mauro Sbragaglia, Eur. Phys. J. E 38, 126 (2015) "Sliding droplets of Xanthan solutions: A joint experimental and numerical study", DOI: 10.1140/epje/i2015-15126-0.

11. François-Gaël Michalec, François G. Schmitt, Sami Souissi, Markus Holzner, Eur. Phys. J. E 38, 108 (2015) "Characterization of intermittency in zooplankton behaviour in turbulence", DOI: 10.1140/epje/i2015-15108-2.

12. Antonio Lasanta, Pablo I. Hurtado, Antonio Prados, Eur. Phys. J. E 39, 35 (2016) "Statistics of the dissipated energy in driven diffusive systems", DOI: 10.1140/epje/i2016-16035-4.

13. Duane Rosenberg, Raffaele Marino, Corentin Herbert, Annick Pouquet, Eur. Phys. J. E 39, 8 (2016) "Variations of characteristic time scales in rotating stratified turbulence using a large parametric numerical study", DOI: 10.1140/epje/i201616008-7.

14. Kartik P. Iyer, Irene Mazzitelli, Fabio Bonaccorso, Annick Pouquet, Luca Biferale, Eur. Phys. J. E 38, 128 (2015) "Rotating turbulence under 'precession-like' perturbation", DOI: 10.1140/epje/i2015-15128-x.

15. Patricio Clark di Leoni, Pablo J. Cobelli, Pablo D. Mininni, Eur. Phys. J. E 38, 136 (2015) "The spatio-temporal spectrum of turbulent flows", DOI: 10.1140/epje/i2015-15136-x.

16. Ganapati Sahoo, Luca Biferale, Eur. Phys. J. E 38, 114 (2015) "Disentangling the triadic interactions in Navier-Stokes equations", DOI: 10.1140/epje/i2015-15114-4.

17. Nicolas Francois, Hua Xia, Horst Punzmann, Michael Shats, Eur. Phys. J. E 38, 106 (2015) "Wave-particle interaction in the Faraday waves", DOI: 10.1140/epje/i2015-15106-4.

18. Michele Buzzicotti, Brendan P. Murray, Luca Biferale, Miguel D. Bustamante, Eur. Phys. J. E 39, 34 (2016) "Phase and precession evolution in the Burgers equation", DOI: 10.1140/epje/i2016-16034-5.

19. Alessandra S. Lanotte, Shiva Kumar Malapaka, Luca Biferale, Eur. Phys. J. E 39, 49 (2016) "On the vortex dynamics in fractal Fourier turbulence", DOI: 10.1140/epje/i2016-16049-x.

Alessandra S. Lanotte, Massimo Cencini, Mauro Sbragaglia, and Luca Biferale 